\title{
La visione della rete: la sfida della didattica integrativa della lingua, della letteratura e del film di lingua straniera
}

\author{
Simona Bartoli Kucher \\ Università di Graz, Austria \\ simona.bartolikucher@uni-graz.at
}

Parallelamente alla realtà culturale e linguistica del $21^{\circ}$ secolo - caratterizzata dalla mescolanza di lingue e culture - anche i nuovi descrittori del Volume Complementare del QCE R (Council of Europe 2018) hanno propagato la necessità del plurilinguismo e delle competenze pluriculturali come obiettivo primario dell'insegnamento delle lingue straniere. Nel seguente intervento, basato su un progetto di didattica cooperativa realizzato con gli studenti di italiano dell'Università di Graz sulla base del paradigma teorico della Transformative Research Action, si intende evidenziare il potenziale dei testi letterari - nati negli interstizi tra lingue e culture - per la formazione degli insegnanti di lingua straniera (Bartoli Kucher 2019). I codici culturali e linguistici plurali dei testi di Carmine Abate e di Laila Wadia sono riusciti a generare e/o a rafforzare negli apprendenti competenze sociali, affettive e interculturali parallelamente alle competenze ricettive e produttive in lingua straniera. I risultati della nostra ricerca empirica sulla ricezione dei testi transculturali da parte degli insegnanti di italiano in formazione ha confermato la nostra ipotesi: i testi letterari transculturali, se ancorati in un approccio didattico pluriculturale e plurilingue (Kramsch 2006; Vedovelli 2015), riescono a sensibilizzare gli apprendenti nei confronti di una società in cui lingue e culture sono in costante contatto. Per questo riteniamo che la didattica integrativa della lingua e della letteratura debba rappresentare uno standard nella formazione degli insegnanti di lingua straniera.

Parole chiave: rete transculturale, scrittura plurale, vivere per addizione, motivazione, letteratura come campo d'azione, didattica cooperativa (cc)BY-SA https://doi.org/10.26493/978-961-293-045-5.119-137

\section{Introduzione e scopo della ricerca}

I testi letterari e i film rappresentano nella didattica delle lingue uno strumento dalle straordinarie potenzialità. Se le storie declinate da questi testi assomigliano a quelle della realtà e mettono al centro codici plurali e plurilingui (Vedovelli 2017) generano una forte motivazione negli apprendenti. Le nostre riflessioni, basate sul progetto «Scritture in viaggio/Schreiben unterwegs/مسافرة كتابات - realizzato a partire dal 2015 all'Istituto di Ro- 
manistica dell'Università di Graz dall'autrice - intendono raccontare il forte impatto motivazionale creato sugli student teachers of language ${ }^{1}$ sia dall'incontro con i testi letterari e filmici di Laila Wadia e di Carmine Abate, che dal dialogo con gli autori. Il progetto ha portato a Graz nel 2015 Sumaya Abdel Qader e Amara Lakhous, Igiaba Scego nel 2017, Wadia nel 2018 e Abate nel 2019, coinvolgendo gli insegnanti in formazione dell'Istituto di Romanistica che, dopo aver letto e didattizzato i testi letterari scelti, hanno realizzato a scuola numerose attività creative nel corso del tirocinio previsto dal curriculum. Sono state le studentesse e gli studenti a presentare gli autori e i testi alla Casa della Letteratura (Literaturhaus) di Graz, ${ }^{2}$ moderando poi all'università la discussione e l'intervista con gli autori, a cui hanno partecipato anche i loro alunni (cfr. Bartoli Kucher 2016, 226-231; Bartoli Kucher 2019, 24-29).

Ci proponiamo di dimostrare che i testi letterari nati negli interstizi tra lingue e culture (Bhabha 1994) rappresentano una carta vincente per la formazione degli insegnanti di lingua straniera.

\section{Metodologia: didattica integrativa della lingua, della letteratura e del film}

Con il diffondersi della diversità culturale e linguistica della nostra società, caratterizzata dalla mescolanza di lingue e culture, anche lo sfondo educativo dell'apprendimento linguistico è cambiato. Secondo Kramsch, nel ventunesimo secolo chi apprende una lingua sa di non doversi confrontare solo con «monolingual native speakers» riconducibili a culture nazionali, ma piuttosto con «multilingual individuals who have grown up in a variety of national, supranational, and ethnic cultures» (Kramsch 2006, 250).

Secondo Bhabha (1994) in questa nuova realtà è pertanto necessario spostarsi dalle specificità di categorie come razza, genere, generazione, situazione istituzionale, ambiente geopolitico e orientamento sessuale. Innovativa e fondamentale è l'intenzione di focalizzare l'attenzione su «those moments or processes that are produced in the articulation of cultural differences» (Bhabha 1994, 2).

La nostra concezione di didattica integrativa della lingua, della letteratura e del film di lingua straniera si basa su una rete di testi connessi dal

\footnotetext{
${ }^{1}$ Questa la definizione degli «insegnanti di lingua in formazione» usata dall'E P O S T L (Newby et al. 2007).

${ }^{2} \mathrm{Si}$ veda https://events.uni-graz.at/de/detail/article/alumni-romanistik-scritture-in -viaggio-schreiben-unterwegs-i-3/.
} 
loro carattere transmediale e dall'uso di codici linguistici plurali (Vattimo e Welsch 1998; Bartoli Kucher 2019). Partiamo dal presupposto che la competenza simbolica debba ampliare la competenza comunicativa e che la prima debba essere nutrita, a tutti i livelli del curriculum di apprendimento linguistico, dall'immaginazione letteraria (Kramsch 2006, 251):

For it is through literature that learners can communicate not only with living others, but also with imagined others and with the other selves they might want to become. Through literature, they can learn the full meaning making potential of language.

In questo paradigma di ricerca è proprio la visione della rete (Welsch 2003, 19) a costituire il termine ombrello sotto il quale si raccolgono i testi nati nei nostri anni negli «in between spaces» (Bhabha 1994, 4).

\section{Dalla competenza comunicativa alla competenza simbolico comunicativa}

Ci occuperemo del romanzo della cantastorie italo indiana Lily-Amber Laila Wadia Amiche per la pelle (2007a) ${ }^{3}$ e dei racconti di Carmine Abate tratti dalle raccolte Il banchetto di nozze e altri sapori (2016) e Vivere per addizione e altri viaggi (2010). Questi testi occupano «spazi di mezzo» perché prendono le mosse da una «international dimension both within the margins of the nation-space and the boundaries in-between nations and peoples» (Bhabha 1994, 4). Sono narrazioni accomunate dall'ibridismo culturale e dal neoplurilinguismo dell'Italia contemporanea, da quel quarto asse dello spazio linguistico italiano in cui, accanto alla lingua standard, accanto ai dialetti e alle lingue minoritarie di antico insediamento, si stanno inserendo le lingue immigrate (Vedovelli 2015, 95).

Questi testi possono diventare molto produttivi sia nella classe di italiano come lingua straniera, che nella classe di italiano come lingua seconda: lo ha dimostrato la ricezione dei testi letterari transculturali italofoni (Kleinhans e Schraderer 2013) da parte degli insegnanti in formazione dell'Istituto di Romanistica dell'università di Graz tra il 2015 e il 2018. La documentazione empirica, basata sul già citato progetto Scritture in viaggio nel Mediterraneo (Bartoli Kucher 2019, 95-126), racconta il forte impatto motivazionale creato sugli insegnanti in formazione sia dall'incontro con i testi letterari e filmici, che dal dialogo con gli autori transculturali. I testi

${ }^{3}$ Del romanzo è stata realizzata nel 2017 anche la trasposizione cinematografica, alla sceneggiatura ha collaborato la stessa Wadia (Babylon Sisters 2017). 
nati negli interstizi tra lingue e culture dovrebbero perciò rappresentare uno standard nella formazione degli insegnanti di lingua straniera: questa è l'ipotesi da cui partiamo.

\section{Tra teoria e pratica: dal language al cultural awareness}

Già nel 2004 Heidi Rösch rifletteva sul ruolo di mediazione culturale assunto dai testi della letteratura transculturale ${ }^{4}$ che spesso hanno dei tratti autobiografici. Per quanto nel discorso della ricerca il carattere autobiografico di questo tipo di narrazioni abbia inizialmente fatto dubitare del loro valore estetico - rimandando piuttosto al loro carattere documentario (Rösch 2004, 89-109) - riteniamo che proprio questi testi rappresentino un fondamentale punto di partenza nella formazione all'educazione interculturale, sia nell'apprendimento scolastico, che in quello universitario (Bredella 2008, 14):

The teaching of literature has to highlight the readers' personal and emotional involvement with the text and how they relate the world of the literary text to their own.

Ne dà esempio questo brano tratto da Il banchetto di nozze e altri sapori di Carmine Abate ${ }^{5}(2016,45)$ :

Avevo undici anni e frequentavo la scuola media di San Nicola dell'Alto. Quell'inverno il freddo era intenso anche da noi, il vento soffiava umido e gelido, a volte nevicava. Buon segno.

Mio padre trascorreva l'inverno in paese perché il cantiere stradale, in cui lavorava ad Amburgo, chiudeva grazie a Schlechtwetter e riapriva, di solito, ai primi di marzo.

Anche se non conoscevo il significato, da bambino amavo la parola Schlechtwetter perché l'associavo al periodo più zuccherigno dell'anno, quello della mia famiglia unita. E con il passare del tempo mi ero convinto che Schlechtwetter fosse un padrone buono, che mandava un suo fatigatòre a svernare in paese. Perciò restai deluso quando mio padre me ne tradusse il senso, dopo avermi preso in giro: «Sei

\footnotetext{
${ }^{4}$ Per quanto riguarda il concetto di transculturalità ci richiamiamo alle riflessioni di Welsch secondo cui il concetto di transculturalità corrisponde, meglio di quello precedentemente diffuso di interculturalità, all'intreccio, alla compenetrazione, all'ibridazione di culture della società contemporanea (Welsch 1992; 2010; 2012; 2017).

${ }^{5}$ Il titolo del racconto è «Il sapore della cuntentizza».
} 
proprio bala bala, come i tedeschi chiamano chi tiene la capa un pochicello sballata. Schlechtwetter non è una persona, ma moti i lig, il cattivo tempo».

Allora pregai Sant'Antonio che in Germania il gelo, la neve, la pioggia non finissero mai e che mio padre non dovesse ripartire, perché tanto con quel tempaccio perenne non avrebbe potuto svolgere il suo lavoro: incatramare le strade.

Il testo ha una struttura sintattica semplice e per questo può essere proposto sia in una classe di italiano come lingua materna, che in una classe di italiano come lingua straniera o seconda per introdurre ambiti tematici come la famiglia e gli amici, la scuola e la formazione, le esperienze personali, le vicende vissute e gli incontri, previsti di solito dai piani di studio delle lingue moderne. ${ }^{6}$

La prima espressione in corsivo - Schlechtwetter - è una parola della lingua del pane che il padre di Abate ha dovuto imparare quando si è trasferito ad Amburgo, spinto dal ricatto del lavoro: la necessità di lavorare per poter far studiare i propri figli perché non fossero a loro volta costretti a emigrare. All'autore nativo di Carfizzi interessa sottolineare che per molti lavoratori meridionali la scelta di emigrare è stata dettata dalla speranza di rendere i propri figli liberi dalla costrizione a cui loro stessi invece sono stati obbligati. $^{7}$

Moti i lig è invece un termine dell'arbëresh, la lingua madre dell'autore la lingua dei suoi antenati fuggiti alla fine del' 400 dai Balcani per non dover sottostare all'Impero ottomano - l'unica da lui parlata fino all'esperienza scolastica. Altre parole provengono dal dialetto calabrese (fatigàtore, la capa un pochicello sballata) o amburghese (bala bala), a sottolineare l'ampio spazio della lingua dell'autore di Carfizzi, rafforzato anche da "parole nuove che si impigliano nella pagina» come esche che declinano le emozioni (p.e. il periodo più zuccherigno dell'anno). ${ }^{8}$

Con Il banchetto di nozze e altri sapori (Abate 2016) è riuscito a imbandire un banchetto plurilingue dalla Calabria della sua infanzia fino ad Amburgo

${ }^{6}$ Si tratta dei temi della comunicazione previsti sia dal QCE R 2002 (Consiglio d'Europa 2002, 65), che dai documenti di politica educativa del sistema scolastico di ambito germanofono.

${ }^{7}$ "Ce ne siamo andati da casa perché non si guadagnava abbastanza per costruire un avvenire ai figli nostri e non perché eravamo dei morti di fame, come credono gli ignoranti razzisti qui in Germania» (Abate 2016, 93).

${ }^{8}$ Così Abate ha definito i suoi neologismi in occasione dell'incontro del 14/12/2019 organizzato a Bressanone dal Comitato Educazione Permanente Evviva, dal Circolo culturale e ricreativo calabrese e della Biblioteca civica di Bressanone. 
dove a 16 anni - su invito del padre - è andato a lavorare per imparare «come si mangia il pane», scegliendo poi di trasferirsi in Trentino come tappa geografica centrale tra i luoghi della sua esistenza. Un banchetto che ripercorre i punti d'arrivo della sua vita e della sua scrittura, declinati lungo il filo conduttore del «vivere per addizione» (Abate 2010). L'autore ha fatto sua questa metafora adattandola alla propria vita e applicandola alla sua scrittura. Vivere per addizione è secondo noi il termine ombrello per gli In Between Spaces da cui siamo partiti, una possibilità concreta per rendere operativa l'educazione transculturale a scuola o all'università: per trasformare in ricchezza la ferita dell'emigrazione. L'emigrazione è un fenomeno strutturale della nostra società, noto a tante studentesse e studenti delle nostre scuole e università per averlo vissuto sulla propria pelle o su quella dei propri genitori, noto anche a tutti gli altri perché presente nella realtà quotidiana.

Abbiamo letto i testi di Abate parallelamente a quelli della narrastorie italiana e indiana Laila Wadia, nata a Bombay, poliglotta fin dalla nascita, la cui prima lingua di scrittura è stata l'inglese per diventare poi l'italiano ${ }^{9}$ quando dopo i venti anni si è trasferita a Trieste.

Le quattro Amiche per la pelle, che danno il titolo al primo romanzo di Wadia (2007a), vivono senza contratto d'affitto in una palazzina fatiscente di Via Ungaretti, in uno spazio plurilingue in cui sono presenti tutte le lingue dei contesti di immigrazione (Vedovelli 2017, 20): il triestino dell'unico inquilino italiano, le lingue straniere di comunicazione internazionale, le varietà interlinguistiche di italiano sviluppate dalla famiglia cinese di Bocciolo di Rosa, dalla famiglia albanese di Lule, dalla famiglia bosniaca di Marinka, come dalla famiglia indiana di Shanti, l'io narrante della storia. Il lettore attento intravede una precisa struttura della funzione simbolica e identitaria delle lingue, anche dei dialetti e della lingua di contatto che evidenzia la marginalità delle famiglie degli immigrati.

\section{Tra teoria e pratica: il neoplurilinguismo}

Abate è cresciuto analfabeta nella sua madrelingua. Dopo aver raggiunto il padre in Germania dove ha trascorso un periodo di lavoro, ha avvertito l'e-

\footnotetext{
${ }^{9}$ Come ha precisato Igiaba Scego sull'Internazionale, in occasione del conferimento del Premio Strega 2018 a Helena Janeczek, scrittrice nata in Polonia e cresciuta in Germania, che pure ha scelto l'italiano come lingua di scrittura: «Non è una scelta ovvia, quella di scrivere in italiano. Si sceglie un orizzonte, una musicalità, un percorso di vita. Di certo non si sceglie il mercato dei libri che contano, che ormai parla solo l'inglese di Manhattan e di Soho. È una scelta politica e letteraria» (Scego 2019).
} 
sigenza di scrivere per denunciare l'ingiustizia di non aver potuto godere, nella sua infanzia, della vicinanza di entrambi i genitori in carne ed ossa e ha scelto di scrivere in italiano (Abate 2019). Il padre di Carmine in Germania aveva imparato il germanese, lingua ibrida fatta di parole tedesche, parole italiane tedeschizzate, parole tedesche italianizzate, parole arbëresh e dialettali ibridate. Per questo Abate si definisce un «transfuga linguistico» (Abate 2019), una figura ibrida come la lingua dei germanesi, ampliata dai vari strati culturali e linguistici di cui lui stesso ha fatto esperienza, imparando a considerarli di volta in volta un arricchimento.

Anche la narrastorie Wadia ha scelto l'italiano probabilmente come «lingua-distanza» (Abate 2019) capace - come scrive Abate - «di filtrare le [proprie] esperienze», per riuscire a «scrivere su una materia scottante come la migranza» senza cadere nella retorica della nostalgia (Abate 2019). ${ }^{10}$ Nella cultura indiana la funzione del narrastorie è quella di portare le storie da un villaggio all'altro per far conoscere realtà diverse: «una persona che non racconta agli altri, ma racconta con gli altri» (Wadia 2016).

\section{Modelli di incontro e di interazione}

Ci immergeremo nei testi narrativi indicati, seguendo il filo delle diverse possibili risposte al percorso di inserimento sociale dei singoli personaggi, mettendo in evidenza che le storie dei protagonisti di questi testi letterari rispecchiano le storie degli immigrati della nostra società.

Spesso lo sguardo su chi è diverso perché straniero o migrante è influenzato da stereotipi (Bolatti et al. 2004), connotato dal pregiudizio dell'inferiorità. Per le donne multiculturali del condominio di via Ungaretti l'interazione sociale comincia attraverso l'apprendimento della lingua. Shanti, Marinka, Lule e Bocciolo di Rosa sanno che la lingua è elemento fondamentale di identità e di interazione. Il lettore lo apprende dalla voce narrante di Shanti (Wadia 2007a, 47):

La cosa che mi colpisce di più di questo piccolo e perfetto mondo multiculturale che siamo riusciti a creare in via Ungaretti 25 è l'idioma in cui ci confidiamo le cose. Provenienti dai quattro angoli del mondo, ci troviamo in questo stretto lembo di terra, [...] a comprenderci in una lingua adottiva. È uno sforzo che abbiamo fatto noi, non per semplice

\footnotetext{
${ }^{10}$ «Penso che gli scrittori stranieri che scrivono in italiano utilizzino anche loro l'italiano come lingua-distanza capace di filtrare le loro esperienze, perché altrimenti scivolerebbero [...] un po' nella retorica, un po' nella nostalgia scontata» (Abate 2019, 3-4).
} 
necessità, ma per la voglia di diventare amiche, di poter andare oltre un semplice «Buongiorno, come stai?» scambiato per le scale.

Le donne di Via Ungaretti, consapevoli dell'importanza «di abbattere il muro linguistico» (Wadia 2007a), hanno trovato un'ex insegnante di scuola media che insieme ai fondamenti di grammatica e di fonologia, cerca d'inculcare alle sue alunne anche l'importanza della libertà e dell'emancipazione femminile, valori alle volte non noti «a chi è nato altrove» (Wadia 2007a, 48).

Nei testi di Abate il ruolo dell' istruzione - come abbiamo già sottolineato - è per i padri prioritario per l'avvenire del figlio (Abate 2016, 69-70):

Devi studiare, bir. E poi farai il culo a coppa a tutti quei signorotti che ci hanno costretto a emigrare, che ci sfruttano l'anima mi aveva detto prima di partire. Non solo. Aveva aggiunto che dovevo essere il primo della classe, il primo della nostra famiglia a diventare studiato, il primo nella vita. Un po' troppo per un ragazzino nato in una casa come la mia e cresciuto in un paese povero, per giunta schiacciato dalle ingiustizie e dallo sfruttamento di pochi notabili. Mio padre sapeva che le sue esortazioni non mi avrebbero lasciato indifferente e perciò, se possibile, da quel giorno studiai più di prima, fino a sera tardi.

Nella lingua dei personaggi raccontati dai nostri autori, sono fondamentali temi e termini dai contenuti identitari. Al contrario dei loro mariti, le donne di via Ungaretti compiono un percorso di interazione che si manifesta sia attraverso l'apprendimento della lingua, che attraverso l'abbigliamento. L'io narrante ha rinunciato per.es. a portare il sari a Trieste: «io ora indosso quasi sempre i pantaloni [...] invece degli abiti indiani che usavo prima. [...] Ashok [il marito di Shanti] ha detto solo che i pantaloni sono più pratici in questo clima così freddo" (Wadia 2007a, 33), anche se quell'abito continua a darle sicurezza, un senso di stabilità, qualcosa su cui contare (Abate 2016, 118).

Uno sguardo critico, alimentato da testi come quelli presentati, mette in grado gli apprendenti - nelle classi multiculturali e plurilingue della nostra società patchwork ${ }^{11}$ - di riflettere sul concetto di identità: il confronto tra le

\footnotetext{
${ }^{11}$ Secondo il rapporto della Caritas sull'immigrazione (Fondazione Migrantes 2019), i bambini nati da genitori stranieri rappresentano il 14,9\% del totale delle nascite; questi dati hanno portato l'Italia nel 2016 al primo posto tra i paesi U E per numero di acquisizioni di cittadinanza.
} 
pareti scolastiche con lingue e culture, specchio delle lingue e culture della società, può diventare punto di partenza per favorire l'identità linguistica $\mathrm{e}$ culturale dei singoli (Wildemann e Hoodgarzadeh 2013, 7), potenziando al contempo la conoscenza del modo di vivere di culture diverse dalla propria e creando ponti a partire dalla propria esperienza e conoscenza della realtà.

\section{Ruolo identitario del cibo}

Il cibo ha per i personaggi di Wadia e di Abate un forte riferimento identitario. Accettando e conoscendo il cibo del paese in cui si vive, si può contribuire all'interazione con la cultura di accoglienza «senza per questo perdere la specificità della propria identità originaria» (Wadia 2016, 3). Marin$\mathrm{ka}$, arrivata col marito in Italia dopo la traumatica esperienza della guerra nei Balcani, più di tutte le altre amiche di Via Ungaretti vuole «dimenticare il passato e diventare italiana». Anche per questo prepara per tutte la jota, tipico piatto triestino: «Se vogliamo integrarci, dobbiamo iniziare mangiando il loro cibo, non credete?» (Wadia 2007a, 40): ${ }^{12}$

Vi dico una cosa, voi non volete veramente far parte di questa società. Voi volete essere diversi. Vi crogiolate nel vostro stato di miserevoli stranieri! Vi ostinate ad aggrapparvi al vostro passato, a un tempo e un paese che non esistono più al di fuori della vostra fantasia. Che senso ha prendere lezioni di italiano? Spaccarvi la testa per imparare le coniugazioni dei verbi? Sforzarvi di leggere I promessi sposi e andare al cinema a vedere Il postino? Se rifiutate le basi di una cultura, la sua cucina, cioè, se non riuscite a mandare giù nemmeno un boccone di jota, come intendete digerire la vita in questo paese?

Parallelamente nei racconti di Abate il cibo rappresenta sia un forte elemento di identità che una possibilità di accettazione della pluralità delle culture, quindi, di interazione. Il cuoco di Arbëria, personaggio di molti testi, rappresenta la possibilità di contribuire all'interazione con la cultura di accoglienza proprio attraverso la contaminazione dei sapori (Abate 2016, 102):

Ogni luogo è un sapore. Chissà che palato ricco di gusti ti farai vivendo in tanti posti diversi. L'importante è che li aggiungi ai sapori

\footnotetext{
${ }^{12}$ Il tema del cibo come collante identitario si trova anche in altri testi di Wadia: si veda il racconto "Curry di pollo» (Wadia 2005) e la raccolta di racconti Mondo pentola (Wadia 2007b).
} 
della nostra terra, di quelli siamo fatti nel profondo, della sua scorza odoriamo, anche se viviamo altrove.

Un modo per favorire l'incontro, a scuola o all'università, è quello di evidenziare la biografia linguistica e la storia degli apprendenti (Fornol 2012, 161-182). Della lingua parlata nella propria famiglia d'origine si trattengono per lo più «termini dai contenuti identitari (riguardanti il cibo, la religione, il sistema parentale, i codici sessuali)» (Brogi 2011): i testi di Wadia e Abate ne rappresentano un esempio.

Lavorare con questi testi servirà ad alcuni a riconoscersi nelle esperienze raccontate in lingua straniera; altri si sentiranno invitati a reagire, a cambiare prospettiva. Per tutti i lettori apprendenti questi testi offriranno la possibilità di una riflessione critica.

\section{Risposte al percorso di inserimento sociale}

Come abbiamo già visto, $\mathrm{i}$ testi proposti rispecchiano risposte diverse al percorso di inserimento nella nuova società da parte degli immigrati. Mentre le amiche di Via Ungaretti fanno di tutto per imparare l'italiano e riescono a capirsi nonostante le diversità, i loro mariti tendono a frequentare solo persone del proprio paese, isolandosi (Wadia 2007a, 71):

Noi donne abbiamo tessuto una fitta trama d'amicizia, ma purtroppo i nostri mariti non si conoscono tanto bene perché sono quasi sempre al lavoro e nel poco tempo libero di cui dispongono preferiscono stare a casa oppure con i propri connazionali.

Come spiega Zagrebelsy (2019) gli immigrati reagiscono spesso alle difficoltà di inserimento sociale con la paura dell'altro e quindi si isolano: è così che nascono i ghetti. Talvolta i migranti considerano invece la propria identità di partenza come un ostacolo e si assimilano alla cultura del paese d'arrivo.

Nel microcosmo multiculturale di Via Ungaretti è l'io narrante che, vivendo e apprezzando la pluralità delle culture, riesce invece (Lazzari 2014, 7) «ad incarnare [...] l'ideale di identità ibrida o multipla» (Wadia 2007a, 119):

Per anni e anni io e le mie vicine abbiamo cercato di mimetizzarci, ma ora ho voglia di far vedere chi sono davvero: Shanti Kumar, una donna quasi trentenne dell'India centrale, tenera ma tenace, con un suo lavoro indipendente da babysitter, che parla benino l'italiano e 
ama cucinare il curry. Sono diventata una specie di ibrido culturale e linguistico, ma il mio cuore è sempre rimasto in un sari.

Anche la fiaba multiculturale della trasposizione cinematografica del romanzo di Wadia - intitolato Babylon Sisters (2017) - comincia dallo scontro tra culture diverse nella palazzina di Via Ungaretti, per concludersi con un modello positivo di interazione realizzata in musica. All'ingiunzione di sfratto con cui il film comincia, le donne del condominio reagiscono aprendo insieme una scuola di ballo con cui risolveranno in parte i loro problemi economici. $^{13}$

\section{Risultati del progetto: rendere operativa l'educazione transculturale}

Non c'è neanche bisogno di sottolineare che le sequenze dei testi letterari e/o del film possano offrire, in una classe di italiano come lingua straniera o come lingua seconda, occasioni autentiche di produzione orale e di produzione scritta.

La didattica integrativa della lingua, della letteratura e del cinema è una scienza che fa dell'integrazione tra prospettive linguistico applicate e semiotiche uno dei fondamenti della propria identità (Bartoli Kucher 2019). Il tratto integrazione indica l'ampliamento dell'oggetto a una pluralità di linguaggi e lingue. Il motore di questo paradigma di didattica delle lingue moderne sta nei testi prodotti negli spazi di mezzo, caratterizzati dalla contaminazione di lingue e culture. Leggendo e lavorando con questi testi, gli apprendenti di una lingua straniera diventano attanti (Fäcke 2010). Il plurilinguismo di questi testi, che rispecchia la pluralità di lingue e culture - diventata «increasingly relevant over the past twenty year» (Council of Europe 2018, 23) - ci pare ideale per realizzare il cambio di paradigma educativo auspicato dal Companion Volume (Council of Europe 2018), i cui nuovi descrittori declinano da un lato la possibilità di «esprimere una reazione personale» ai testi creativi già a partire dal livello dei principianti, dall'altro propongono di sviluppare gli ambiti della «mediation» e delle «plurilingual and pluricultural competence» tralasciati dalla versione originale del QCE R (Consiglio d'Europa 2002).

Cercheremo di offrire nel testo che segue degli spunti operativi specifici, basati sulla nostra esperienza didattica e sull'incontro degli studenti dell'Università di Graz con Wadia e Abate. I loro testi narrativi transcul-

${ }^{13} \mathrm{Si}$ veda in proposito Bartoli Kucher (2020, 513-514). 
Tabella 1 Choice board

\begin{tabular}{|c|c|c|}
\hline $\begin{array}{l}\text { Mettersi nei panni di qualcuno } \\
\text { Mettiti nei panni di Shanti. } \\
\text { Vuoi convincere in italiano tuo } \\
\text { marito dell'importanza che } \\
\text { vostra figlia frequenti amici e } \\
\text { conoscenti italiani. Mettiti nei } \\
\text { panni del figlio/della figlia del } \\
\text { «Cuoco di Arbëria» e raccon- } \\
\text { ta ai tuoi amici/che la storia di } \\
\text { tuo padre. }\end{array}$ & $\begin{array}{l}\text { Dialogo } \\
\text { Mettetevi nei panni delle } \\
\text { "amiche per la pelle» e discute- } \\
\text { te sul modo migliore per con- } \\
\text { vincere il padrone di casa a } \\
\text { non darvi lo sfratto. Mettete- } \\
\text { vi nei panni degli amici di Car- } \\
\text { mine e raccontate della vostra } \\
\text { famiglia. } \\
\text { A2 Can describe a character's } \\
\text { feelings and explain the reasons } \\
\text { for them. }\end{array}$ & $\begin{array}{l}\text { Lettera a... } \\
\text { Una delle donne di Via Un- } \\
\text { garetti scrive a nome di tutte } \\
\text { una lettera di ringraziamento } \\
\text { alla maestra di italiano. Scri- } \\
\text { vi che cosa hai imparato, che } \\
\text { cosa ti è piaciuto delle lezioni, } \\
\text { che cosa non ti è piaciuto. }\end{array}$ \\
\hline UVo & & \\
\hline $\begin{array}{l}\text { Trasforma la storia delle ami- } \\
\text { che per la pelle o una delle sto- } \\
\text { rie di Abate in una favola a lie- } \\
\text { to fine. Trasforma la storia } \\
\text { delle amiche per la pelle in un } \\
\text { giallo. }^{2}\end{array}$ & $\begin{array}{l}\text { Immagina di intervistare una } \\
\text { delle amiche di Via Ungaret- } \\
\text { ti/uno dei personaggi di Abate } \\
\text { emigrato in Germania. Chie- } \\
\text { di: come si è sentita/o quan- } \\
\text { do è arrivata/o in Italia/in } \\
\text { Germania, che cosa è cambia- } \\
\text { to dopo i primi anni, che co- } \\
\text { sa cambierebbe nella sua vita } \\
\text { quotidiana. }\end{array}$ & $\begin{array}{l}\text { In gruppi di } 4 \text { mettete in sce- } \\
\text { na una delle situazioni raccon- } \\
\text { tate nel testo e dite se vi è pia- } \\
\text { ciuta. Registratela col telefoni- } \\
\text { no. Alla fine tutte le scenette } \\
\text { vengono presentate al gruppo. } \\
\text { A1 Can use simple words or } \\
\text { phrases to say how a work made } \\
\text { him/her feel. }\end{array}$ \\
\hline \multicolumn{2}{|c|}{$\begin{array}{l}\text { B2 Can relate events in a story, film or play to similar events } \\
\text { he/she has experienced or heard about. }\end{array}$} & $\begin{array}{l}\text { A2 Can say whether he/she liked } \\
\text { a work or not and explain why in } \\
\text { simple language. }\end{array}$ \\
\hline
\end{tabular}

Continua alla pagina seguente

turali rappresentano «corpora importanti per i processi di apprendimento linguistico, proprio perché da un lato declinano forme di espressione descrittive e comunicative, coinvolgendo dall'altro - in base al loro potenziale di autenticità [... ] - il piano emotivo e riflessivo» (Bartoli Kucher 2019, 13).

La nostra concezione di didattica integrativa si basa anche sulle riflessioni di Nussbaum che vede la letteratura come fonte insostituibile per coltivare l'immaginazione, il pensiero critico e l'abilità di capire la condizione degli altri. L’immaginazione narrativa viene infatti definita dalla filosofa americana come (Nussbaum 2010, 96):

[...] the ability to think what it might be like to be in the shoes of a person different from oneself, to be an intelligent reader of that person's story, and to understand the emotions and wishes and desires that someone so placed might have. 
Tabella 1 Continua dalla pagina precedente

\begin{tabular}{|c|c|c|}
\hline $\begin{array}{l}\text { Dialogo interculturale } \\
\text { In dialogo con un/a vostro/a } \\
\text { collega, raccontate un episo- } \\
\text { dio in cui una delle amiche di } \\
\text { Via Ungaretti o uno dei perso- } \\
\text { naggi di Abate si è sentita/o } \\
\text { discriminata/o in Italia o in } \\
\text { Germania. Raccontate episodi } \\
\text { di discriminazione di cui avete } \\
\text { fatto esperienza (o che vi sono } \\
\text { stati raccontati). } \\
\text { B2 Can relate the emotions ex- } \\
\text { perienced by a character in a } \\
\text { work to emotions he/she has } \\
\text { experienced. }\end{array}$ & $\begin{array}{l}\text { Descrizione } \\
\text { Descrivete uno dei personaggi } \\
\text { del testo letterario o del film } \\
\text { di Wadia o di Abate: aspet- } \\
\text { to fisico, carattere, interessi, } \\
\text { prospettive. } \\
\text { B1 Can describe the personality } \\
\text { of a character. }\end{array}$ & $\begin{array}{l}\text { Plurilinguismo e multiculturali- } \\
t \grave{a}^{3} \\
\text { Scegliete uno dei personag- } \\
\text { gi plurilingue dei racconti di } \\
\text { Abate, del romanzo o del film } \\
\text { di Wadia e una sequenza del } \\
\text { romanzo o del film in cui ave- } \\
\text { te individuato più lingue: con- } \\
\text { tinuate la storia in italiano } \\
\text { standard, scrivete il finale } \\
\text { nella vostra lingua d'origine. }\end{array}$ \\
\hline $\begin{array}{l}\text { Dialogo sulle lingue } \\
\text { A coppie: ognuno sceglie una } \\
\text { lingua diversa e prova in italia- } \\
\text { no a convincere l'altro ad im- } \\
\text { pararla; descrivi perché quel- } \\
\text { la lingua è interessante, per- } \\
\text { ché la si dovrebbe imparare, } \\
\text { se è diversa dalle altre lingue } \\
\text { conosciute etc. }\end{array}$ & $\begin{array}{l}\text { Titolo in tutte le lingue } 4 \\
\text { Sii creativoa/o! } \\
\text { Proponi un'attività didattica } \\
\text { per tutta la classe. }\end{array}$ & $\begin{array}{l}\text { Cibo multiculturale } \\
\text { Ognuno di voi propone un } \\
\text { piatto tipico della sua infan- } \\
\text { zia: elenca gli ingredienti, } \\
\text { spiega agli altri come si fa } \\
\text { la ricetta (anche nella pro- } \\
\text { pria lingua madre o nella lin- } \\
\text { gua usata a scuola), il gruppo } \\
\text { sceglie il piatto migliore! }\end{array}$ \\
\hline
\end{tabular}

Note ${ }^{1}$ Le frasi in corsivo di questa tabella, precedute dall'indicazione del livello di competenza previsto dal QCE R, fanno riferimento alla competenza parziale «Expressing a personal reaction to creative texts» del primo sottocapitolo del Companion Volume intitolato «Mediating a Text» (Council of Europe 2018, 116). ${ }^{2}$ Quando si lavora con diversi tipi di testo, è consigliabile analizzarne prima le caratteristiche testuali insieme agli apprendenti. Rimandiamo in proposito a Bartoli Kucher (2013, 95 ss). ${ }^{3} \mathrm{Nel}$ capitolo «Mediating Communication» del Companion Volume del 2018 le varie scale di descrittori prevedono un ruolo più attivo dell'apprendente come mediatore culturale. Al centro sta la competenza sociolinguistica e l'abilità di azione culturale. Come sostiene Burwitz-Melzer le scale risultano però poco precise e difficilmente applicabili (2019, 112-116). 4 Un titolo metaforico come Amiche per la pelle può diventare oggetto di mediazione linguistica e culturale in un approccio ludico a una molteplicità di lingue nell'insegnamento delle lingue straniere, con il coinvolgimento delle rispettive lingue madri. Si veda Bartoli Kucher (2020, 516-517).

Per questo proponiamo di mettere le emozioni degli apprendenti al centro del processo educativo, facilitando così - parallelamente alle competenze ricettive e produttive in lingua straniera - le competenze sociali e affettive e la comprensione interculturale.

Nel choice board (tabella 1) abbiamo raccolto alcune delle proposte emerse dai lavori di gruppo delle studentesse e degli studenti che, dopo aver letto e riflettuto sui testi transculturali letterari e filmici, hanno preparato gli 
allievi e le allieve di alcuni ginnasi sia ad attività creative, che all'incontro con gli autori.

Tutte le attività didattiche si orientano ai nuovi descrittori del CEFR che prevedono per tutti i livelli di competenza la capacità di esprimere una risposta personale ai testi letterari, considerati idonei a suscitare empatia nei lettori apprendenti (Council of Europe 2018, 116). Il paradigma delle emozioni ricorre infatti in ognuno dei livelli di competenza.

Le attività didattiche che riportiamo sono solo esemplificative: possono venire modificate in riferimento al livello di competenza degli apprendenti, e/o in base ai diversi temi scelti di questi o di altri testi letterari o filmici transculturali.

Questo tipo di attività didattiche può migliorare la capacità degli studenti di mettersi nei panni degli altri, relativizzando così il loro punto di vista e sviluppando empatia, sensibilità e partecipazione sociale (Nünning 2007).

La comprensione di storie e il racconto di storie - come quelle da cui siamo partiti - sono pratiche culturali importanti che, concentrandosi su situazioni ed esperienze sia individuali che collettive, permettono di vedere e percepire ciò che le persone di culture diverse pensano e sentono, interpretando i valori e le motivazioni che stanno alla base delle loro azioni (Nünning e Nünning 2007; Hallet e Nünning 2009).

Per questo abbiamo dato priorità a metodi di insegnamento che incoraggino gli studenti a partecipare attivamente alla scoperta, alla sfida, alla riflessione e alla co-costruzione di significato, in particolare per quanto riguarda le riflessioni sul potenziale offerto dai testi per lo sviluppo delle competenze interculturali.

La pratica della lettura è stata condotta in diversi modi: lettura silenziosa, lettura ad alta voce, lettura in piccoli gruppi, lettura interattiva o dialogante, lettura riflessiva, lettura pubblica durante gli incontri con gli autori che hanno dato lettura espressiva dei loro testi. Queste diverse modalità di lettura sono state introdotte con la consapevolezza che, anche all'università, c'è la necessità di una svolta didattica trasformativa attraverso un approccio creativo ai testi letterari.

\section{Conclusioni e prospettive}

Nell'insegnamento delle lingue straniere l'eccessivo orientamento alle competenze introdotto dal QCER ha determinato la marginalizzazione dei testi letterari o filmici, privilegiando invece testi non autentici, creati apposta per introdurre il lessico e le strutture della lingua previsti dal piano 
di studi e dal sillabo. Alla letteratura di lingua straniera non è stata riconosciuta la capacità di sviluppare competenze. L'esclusione della letteratura ha portato a una riduzione delle competenze di lettura e di empatia: lo dimostrano i risultati dello studio OCS E P IS A (Invalsi 2018) che ha avuto come dominio principale proprio la reading literacy.

Come ha dimostrato la nostra documentazione empirica, i testi letterari transculturali incoraggiano non solo la riflessione degli apprendenti su se stessi e sull'apertura alla diversità, ma anche verso la propria diversità linguistica e culturale e il proprio sentimento di appartenenza. La classe di lingua straniera diventa così quello che Kramsch ha definito il Third-Space, il luogo del dialogo in cui le attività comunicative possono diventare cross cultural activities, con lo scopo di una «irriducible confrontation that may change one in the process» (Kramsch 1993, 231; 2009, 233-236).

\section{Riferimenti bibliografici}

Abate, C. 2010. Vivere per addizione e altri viaggi. Milano: Mondadori.

- 2016. Il banchetto di nozze e altri sapori. Milano: Mondadori.

- 2019. «In due mondi.» Digiland. https://digilander.libero.it/ vocidalsilenzio/attiabate.htm.

Babylon Sisters. 2017. Regia di G. Roccati. Zagreb: Antitalent Produkcija; Trieste: TI C Film.

Bartoli Kucher, S. 2013. «Testi letterari e film per capire e raccontare storie: competenza narrativa e competenza interculturale nella didattica delle lingue straniere.» In Contesti di apprendimento di italiano L2 tra teoria e pratica didattica, a cura di M. Rückl, E. Santoro e I. Vedder, 89-10o. Firenze: Cesati.

- 2016. «Un progetto di didattica della letteratura transculturale.» In Sprachen und Kulturen: vermitteln und vernetzen, a cura di M. Rückl, 226232. Münster e New York: Waxmann.

- 2019. Scritture in viaggio nel Mediterraneo: proposte di didattica integrativa tra lingua, letteratura e film. Pisa: Pacini.

- 2020. "Neoplurilinguismo e didattica integrative della lingua, della letteratura e del cinema nella classe di lingua straniera.» Italiano LinguaDue 12 (2): 512-522.

Bhabha, H. 1994. The Location of Culture. Londra: Routledge.

Bolatti, G., R. Bracalenti, P. Braham e S. Gindro. 2004. Dizionario delle diversità: parole e concetti per capire l'emigrazione. Roma: Edup.

Bredella, L. 2008. «What makes Reading Literary Texts Pleasurable and Educationally Significant?» Fremdsprachen Lehren und Lernen 37:12-26.

Brogi, D. 2011. «Smettiamo di chiamarla «letteratura della migrazione»? A proposito di un romanzo di Igiaba Scego (e non solo).» Nazione Indiana, 
23 marzo. Https://www.nazioneindiana.com/2011/o3/23/smettiamo-di -chiamarla-\%C2\%ABletteratura-della-migrazione\% $\mathrm{C}_{2} \% \mathrm{BB} /$.

Burwitz-Melzer, E. 2019. «Plurilinguale und plurikulturelle Kompetenz im C E F R Companion Volume (2018).» In Handbuch Mehrsprachigkeits- und Mehrkulturalitätsdidaktik, a cura di Ch. Fäcke e F.-J. Meißner, 112-116. Tübingen: Narr Francke Attempto.

Consiglio d'Europa. 2002. Quadro comune europeo di riferimento per le lingue: apprendimento insegnamento valutazione. Traduzione dall'inglese di F. Quartapelle e D. Bertocchi. Oxford: La Nuova Italia.

Council of Europe. 2018. "Common European Framework of Reference for Languages: Learning, Teaching, Assessment; Companion Volume with New Descriptors.» Https://rm.coe.int/cefr-companionvolume-with -new-descriptors-2018/1680787989.

Fäcke, Ch. 2010. Fachdidaktik Französisch. Tübingen: Narr Francke Attempto.

Fondazione Migrantes. 2019. XXVIII rapporto immigrazione 2018-2019: non si tratta solo di migranti. Todi: Tau.

Fornol, S. 2012. «Die Förderung der Interkulturalität und des Sprachenselbstbewusstseins mit Hilfe eines Sprachenportfolios - eine Interventionsstudie.» In Mehrsprachigkeit und Unterrichtsforschung, in der Reihe "Papers of Excellence: Ausgewählte Arbeiten aus den Fachdidaktiken«, a cura di D. Elsner e A. Wildemann, 161-182. Aachen: Shaker.

Hallet, W., e A. Nünning. 2009. "Grundlagen und Methoden der Romandidaktik: Kontext, Konzeption und Ziele des Bandes.» In Romandidaktik: Theoretische Grundlagen, Methoden, Lektüreanregungen, a cura di W. Hallet e A. Nünning, 1-12. Trier: WV T.

Kleinhans, M., e Ch. Schraderer. 2013. Transkulturelle italophone Literatur/ Letteratura italofona transculturale. Würzburg: Königshausen \& Neumann.

Kramsch, C. 1993. Context and Culture in Language Teaching. Oxford: Oxford University Press.

- 2006. «From Communicative Competence to Symbolic Competence.» The Modern Language Journal 90 (2): 249-252.

- 2009. "Third Culture and Language Education.» In Contemporary Applied Linguistics, vol. 2, a cura di V. Cook e L. Wei, 233-254. Londra: Continuum.

Lazzari, L. 2014. «Identità e alterità nel romanzo «Amiche per la pelle` di Laila Wadia.» Altrelettere, 26 novembre.

Newby, D., R. Allan, A.-B. Fenner e B. Jones. 2007. European Portfolio for Student Teachers of Language: A Reflection Tool for Language Teacher Education (E P OS TL). Graz: Council of Europe. 
Nünning, A. 2007. «Fremdverstehen und Bildung durch neue Weltansichten: Perspektivenvielfalt, Perspektivenwechsel und Perspektivenübernahme durch Literatur.» In Neue Ansätze und Konzepte der Literatur- und Kulturdidaktik, a cura di W. Hallet e A. Nünning, 123-142. Trier: WVT.

Nünning, V., e A. Nünning. 2007. «Erzählungen verstehen, verständlich erzählen: Dimensionen und Funktionen.» In Literaturunterricht, Kompetenzen und Bildung, a cura di L. Bredella e W. Hallet, 87-106. Trier: WV T.

Nussbaum, M. C. 2010. Not for Profit: Why Democracy Needs the Humanities. Princeton, NJ: Princeton University Press.

Rösch, H. 2004. «Migrationsliteratur als neue Weltliteratur?» Sprachkunst 35:89-109.

Scego, I. 2018. «La vittoria di Helena Janeczek allo Strega va oltre la letteratura.» Internazionale, 6 luglio. https://www.internazionale.it/ opinione/igiaba-scego/2018/o7/o6/helena-janeczek-premio-strega.

Vattimo, G., e W. Welsch, a cura di. 1998. Medien-Welten Wirklichkeiten. Monaco di Baviera: Fink.

Vedovelli, M. 2015. «Fra 40 anni, l'Italia che verrà: lo spazio linguistico e culturale italiano fra lingue immigrate, andamento demografico, ripresa economica.» Italienisch 73 (1): 78-109.

- 2017. «Il cinema e il nuovo spazio linguistico italiano fra immigrazione e emigrazione.» Mosaic: The Journal for Language Teachers 12 (1): 5-32.

Wadia, L. 2005. «Curry di pollo.» In Pecore nere, a cura di G. Kuruvilla, I. Mubiayi, I. Scego e L. Wadia, 39-52. Bari: Laterza.

- 2007a. Amiche per la pelle. Roma: Edizioni e/o.

- 2007b. Mondopentola. Isernia: Cosmo Iannone.

- 2016. Intervista di Lorenzo Mari. http://newitalians.eu/wp-content/ uploads/2016/05/Wadia.pdf.

Welsch, W. 1992. «Transkulturalität: Lebensformen nach der Auflösung der Kulturen.» Information Philosophie 20 (2): 5-20.

- 1999. «Transculturality: The Puzzling Form of Cultures Today.» In Theory, Culture \& Society: Spaces of Culture: City, Nation, World, a cura di M. Featherstone e S. Lash, 195-213. Londra: Sage.

- 2003. «Rolle und Veränderungen der Religion im gegenwärtigen Übergang zu transkulturellen Gesellschaften.» In Religionen in der Pluralität: Ihre Rolle in postmodernen Gesellschaften; Wolfgang Welschs Ansatz in christlicher und islamischer Perspektive, a cura di D. Chr. Siedler, 13-47. Berlino: Alektor.

- 2010. «Was ist eigentlich Transkulturalität?» In Hochschule als transkultureller Raum? Kultur, Bildung und Differenz in der Universität, a cura di L. Darowska, Th. Lüttenberg e C. Machold, 39-66. Bielefeld: Transcript. 
- 2012. «Was ist eigentlich Transkulturalität?» In Kulturen in Bewegung: Beiträge zur Theorie und Praxis der Transkulturalität, a cura di D. Kimmich e S. Schahadat, 25-40. Bielefeld: Transcript.

- 2017. Transkulturalität Realität - Geschichte - Aufgabe. Vienna: New Academic Press.

Wildemann, A., e B. Hoodgarzadeh. 2013. Sprachen und Identitäten. Innsbruck: Studien Verlag.

Zagrebelsky, G. 2019. «Multiculturalismo: intervista a Gustavo Zagrebelsky a margine del convegno «Multiculturalismo e diritti: accomodating diversitys.» Radio Radicale, 22 settembre. Http://www.radioradicale.it/ scheda/262744/multiculturalismo-intervista-a-gustavo-zagrebelsky -a-margine-del-convegno.

Pogled na medmrežje: izziv didaktiki, ki pri pouku integrira jezik, književnost in film v tujem jeziku

Skladno s kulturno in jezikovno stvarnostjo 21. stoletja, ki jo zaznamuje prepletanje jezikov in kultur, novi opisniki spremljevalnega zvezka SE Jo (Svet Evrope 2018) izpostavljajo nujnost razvijanja večjezičnosti in večkulturne zmožnosti kot osnovnih ciljev poučevanja tujih jezikov. Prispevek, ki predstavlja rezultate raziskovanja sodelovalnega učenja, $\mathrm{v}$ katerem so sodelovali študenti italijanščine Univerze v Gradcu, izhaja iz teoretične paradigme Transformativnega raziskovalnega pristopa (Trasformative Research Action) in poudarja potencial književnih besedil, ki so nastali v stiku jezikov in kultur, pri izobraževanju učiteljev tujih jezikov (Bartoli Kucher 2019). Večplastna kulturna in jezikovna koda besedil Carmineja Abateja in Laile Wadia so študentom omogočila razvoj socialne, čustvene in medkulturne zmožnosti sočasno z razvojem sprejemalnih in tvorbenih zmožnosti v tujem jeziku. Rezultati empirične raziskave o recepciji transkulturnih besedil med študenti, bodočimi učitelji italijanščine, so potrdili izhodiščno hipotezo raziskave, in sicer, da transkulturna literarna besedila v povezavi $z$ večkulturnim in večjezičnim didaktičnim pristopom (Kramsch 2006; Vedovelli 2015) omogočajo razvoj občutljivosti študentov do družbe, v kateri se jeziki in kulture stalno prepletajo. Didaktika, ki integrira jezik in književnost, mora zato postati eden od standardov v izobraževanju učiteljev tujih jezikov.

Ključne besede: transkulturna mreža, pluralno besedilo, povezovalno življenje, motivacija, literatura kot polje delovanja, sodelovalno učenje

\section{Viewpoint on the Internet: Challenges of a Didactics Integrating} Language, Literature and Film in a Foreign Language

In parallel to cultural and linguistic reality of the 21st Century, characterized by intertwinig of languages and cultures, new descriptors pubblished in the Companion Volume to the CEFR (Council of Europe 2018) disseminate the 
need for a plurilinguism and pluricultural competences as primary objective in learning and teaching foreign languages. The paper presents a project on cooperative learning conducted with students of Italian at the University of Graz, based on the Transformative Research Action theoretical paradigm. Research results underline the potential of literary texts, written in contact of languages and cultures, for the education of foreign language teachers (Bartoli Kucher 2019). Plural cultural and linguistic codes in texts written by Carmine Abate and Laila Wadia were able to generate and/or enhance students' social, affective, and intercultural competences, simultaneously with receptive and productive competences in a foreign language. Research results on the reception of transcultural texts by pre-service teacher trainees have confirmed the research hypothesis, stating that literary transcultural texts, if ancored to a pluricultural and plurilingual didactic approach (Kramsch 2006; Vedovelli 2015) have the potential to sensitize students to a society in which languages and cultures are constantly in contact. A didactic approach integrating language and literature should thus represent a standard in foreign language teacher training.

Key words: transcultural network, plural writing, living by addition, motivation, literature as a field of action, cooperative didactics 